\title{
La Gobernanza de la Seguridad en Iberoamérica
}

\section{The Governance of Security in Iberoamerica}

\author{
Jorge M. Vega ${ }^{1}$ \\ Universidad del Salvador (Argentina) \\ Nicolás Comini ${ }^{2}$ \\ Universidad del Salvador (Argentina)
}

Recibido: 04-02-17

Aprobado: 24-03-17

\section{Resumen}

Luego de más de 25 años de historia el sistema iberoamericano atraviesa un proceso de consolidación institucional signado por la ininterrumpida concreción de cumbres políticas, la proliferación de organismos especializados y la ejecución de programas específicos de cooperación. No obstante ello, un asunto de reconocida trascendencia internacional y regional como es la seguridad no ha recibido aún un tratamiento comprehensivo y continuado, limitándose su recepción a iniciativas de alcance parcial o declaraciones no vinculantes. A pesar de las perennes diferencias de contexto estratégico, esquemas subregionales de cooperación y prioridades de agenda a ambos lados del Atlántico, la gestión de la seguridad en tanto política pública representa un necesario eje de trabajo para los Estados iberoamericanos. En consecuencia, el presente documento tiene por objeto fundamentar la importancia y pertinencia temporal de su inclusión en los planes de acción del sistema, además de esbozar posibles líneas de cooperación técnica en el tema.

\footnotetext{
${ }^{1}$ (jorge.vega@usal.edu.ar) Licenciado en Relaciones Internacionales, Abogado y Máster en Dirección y Gestión Pública. Docente e investigador, especialista en políticas públicas de seguridad y defensa. Entre sus obras destacan: "Planeamiento de la Defensa en Argentina: Modelo y Perspectivas" (2016)

(http://www.ieee.es/publicaciones-new/documentos-de-opinion/2016/DIEEEO40-2016.html);

"El Planeamiento de la Defensa en España 2008-2015: Análisis de Política Pública" (2015)

(http://www.seguridadinternacional.es/?q=es/content/el-planeamiento-de-la-defensa-enespa\%C3\%B1-2008-2015-an\%C3\%A1lisis-de-pol\%C3\%ADtica-p\%C3\%BAblica).

2 (nicolas.comini@usal.edu.ar) Doctor en Ciencias Sociales, magister en Integración Latinoamericana y licenciado en Relaciones Internacionales. Director de la licenciatura y la maestría en Relaciones Internacionales de la Universidad del Salvador, Buenos Aires. Especialista en cooperación regional. Entre sus obras destacan, Comini, N. and Stola, I. (Comp.) (2016), Políticas Públicas Regionales. Un abordaje sectorial de la integración latinoamericana, Untref-Sciencies Po, Buenos Aires: Eduntref; Comini, N., (2016), suRamericanizados. La integración regional desde la Alianza al kirchnerismo, Ediciones Universidad del Salvador.
} 
Palabras-clave: Gobernanza, cooperación, políticas públicas, seguridad, defensa, Iberoamérica.

\begin{abstract}
After more than 25 years of history the Ibero-American system is undergoing a process of institutional consolidation affected by the uninterrupted realization of political summits, the proliferation of specialized agencies and the execution of specific cooperation programs. Nevertheless, a matter of international and regional importance such as security has not yet received a comprehensive and continuous treatment, finding limited its reception to initiatives of partial scope and non-binding declarations. Despite the differences in strategic context, sub-regional cooperation schemes and agendas on both sides of the Atlantic, security policy management represents a necessary axis of work for IberoAmerican States. Therefore, the purpose of this article is to substantiate the importance and timeliness of its inclusion in the system's action plans, as well as to outline possible lines of technical cooperation on the subject.
\end{abstract}

Key-words: Governance, Cooperation, Public Policies, Security, Defence, Iberoamerica.

\title{
1. Introducción
}

Iberoamérica no es solo una construcción histórico-geográfica sino también una entidad política dotada de una arquitectura propia de cooperación en constante expansión. De hecho, al sistema iberoamericano lo componen actualmente diversos organismos internacionales focalizados en áreas puntuales de políticas públicas como educación, ciencia, cultura y juventud. La ampliación de su plataforma de trabajo ha sido gradual y sostenida, al igual que la consolidación de su presencia en cada país miembro.

A pesar de su creciente trascendencia en la agenda política interna de sus integrantes, la seguridad ocupa aun un incipiente rol dentro de las prioridades de análisis y cooperación del sistema. Sus antecedentes de intervención se limitan a cumbres y foros de alcance parcial (judicial, vial y local) y a declaraciones generales sobre terrorismo y crimen organizado. Sólo el Comunicado Especial sobre Seguridad Ciudadana y Seguridad Pública (2011) constituye una referencia de consideración.

Este estado embrionario de institucionalidad en materia de seguridad se explica por distintos factores, entre los que se destacan la evidente diversidad de contextos estratégicos presente a ambos lados del Atlántico (sumada a las diferencias dentro de América Latina) y la proliferación de instancias 
competentes de cooperación exclusivamente subregionales. A ello debe añadirse la carencia de una red académica específicamente focalizada en la búsqueda de caminos concretos para la generación de tales políticas públicas regionales.

No obstante, en el último decenio se generó un común denominador de necesidades técnicas sobre gestión de la seguridad en tanto política pública que representa una insoslayable ventana de oportunidad para diversificar los canales ya existentes de cooperación. Tales demandas surgieron en el marco de procesos nacionales de reforma del sector seguridad gestados para vigorizar las capacidades públicas de conducción de un área central aunque postergada en las agendas de modernización post recuperación de la democracia.

Dicho perfil de demandas, vinculado esencialmente al diseño de políticas púbicas superadoras, se encuentra perfectamente alineado con las actuales prioridades programáticas del sistema iberoamericano. En este sentido, su énfasis está puesto en la generación de instancias de cooperación técnica en problemáticas comunes como la modernización de la administración pública, la implementación de una gestión basada en resultados y la profesionalización de los servidores públicos.

Una iniciativa de esta naturaleza aportaría a su vez a concretar la declarada pretensión del sistema de contribuir con el desarrollo socioeconómico de la región a través del fortalecimiento de la propia cooperación iberoamericana y de la modalidad de cooperación Sur-Sur, objetivos que pueden cobrar incluso una renovada trascendencia en el corto plazo a partir de los posicionamientos que adopten España y América Latina -que, de por sí, evidencia profundas diferencias en su interior- ante las nuevas definiciones en política exterior de la Administración Trump en Estados Unidos de América.

Ante este panorama, el presente artículo tiene por objetivo principal fundamentar la conveniencia y pertinencia temporal de instalar la cuestión de la política de seguridad (entendida en un sentido amplio) como tema de análisis permanente en el sistema iberoamericano. Adicionalmente, procura identificar ejes y líneas de acción que permitan diseñar un plan inicial de cooperación técnica en el área, inspirando a su vez la generación de nuevas investigaciones y proyectos académicos especializados.

Para dar cuenta de tales objetivos, en primer lugar, se especificará el alcance de los conceptos de gobernanza y seguridad para este trabajo y se reseñarán las particularidades de Iberoamérica como sujeto excluyente de análisis. Posteriormente, se describirá el estado de desarrollo institucional del sistema iberoamericano y reseñarán los rasgos centrales de la agenda de seguridad de la región. Para finalizar, el estudio incluirá un esbozo de potenciales perspectivas de cooperación. 


\section{Iberoamérica, gobernanza y seguridad}

Describir la gobernanza de la seguridad en Iberoamérica requiere previamente especificar el alcance de los tres principales conceptos que integran dicha categoría analítica: gobernanza, seguridad e Iberoamérica. De este modo, se realizará una mención sobre las particularidades de la región como unidad de análisis y una reseña del marco teórico asociado a los conceptos de gobernanza y seguridad, especificando sobre este último el empleo puntual que presenta en América Latina y España.

Iberoamérica constituye una idea que, aunque polisémica y problemática, posee características presentes transversalmente en las interpretaciones emergentes en torno a ella: geográficas, culturales, históricas, económico-financieras, políticas y lingüísticas. La problematización sobre dicha cuestión se remonta a principios del siglo XIX, siendo Monteagudo uno de los pioneros en hacerlo en 1824 a través de su "Ensayo sobre la necesidad de una federación general entre los Estados Hispanoamericanos y Plan de su Organización" (Magariños 2005).

Para algunos autores (Seitz 2010) se trata de una propuesta a partir de la cual "se reacciona de modo conjunto (Hispanoamérica/Iberoamérica) frente a algún peligro externo considerado" y que se distingue de otras alternativas como la latinoamericana y la panamericana (que incluye a los demás países del Caribe, Centro y Norteamérica). En este sentido, para Morán López (1993) "la definición de Iberoamérica no es un sinónimo de Latinoamérica, meramente una definición cultural, sino que es una definición jurídico-política en términos de relación internacional".

Aunque los actores que la integran se han proyectado histórica y simultáneamente a escenarios subregionales, regionales e incluso mundiales, referirse a Iberoamérica implica concentrarse fundamentalmente en aquellos países y organismos internacionales que conforman la denominada Comunidad Iberoamericana. Debe entenderse así como una unidad pero también como elementos disociados, es decir, como la conjunción de 22 Estados soberanos $^{3}$ conformados por diferentes comunidades que conviven en su seno.

En relación a la gobernanza, a pesar de sus múltiples aplicaciones y significados existe un acuerdo básico respecto de su referencia a formas de gobierno más cooperativas en las que se flexibilizan los tradicionales límites entre lo público y lo privado (Natera Peral 2005: 55). Siguiendo a dicho autor, se la define como un "nuevo estilo de gobierno distinto al de regulación jerárquica del Estado, pero también al del mercado, caracterizado por un mayor grado de interacción y de cooperación entre el Estado y los actores no estatales" (2005: 55).

\footnotetext{
${ }^{3}$ Andorra, Argentina, Bolivia, Brasil, Colombia, Costa Rica, Cuba, Chile, República Dominicana Ecuador, El Salvador, España, Guatemala, Honduras, México, Nicaragua, Panamá, Paraguay, Perú, Portugal, Uruguay y Venezuela.
} 
Desde una perspectiva histórica, gobernanza y gobernabilidad son conceptos interrelacionados derivados de la noción de ingobernabilidad, acuñada por la Comisión Trilateral (1975) para describir la realidad política de Estados Unidos, Europa Occidental y Japón durante la década de 1970, signada por una sobrecarga del estado de bienestar, es decir, por la brecha creciente entre demandas sociales en expansión y Estados faltos de recursos, autoridad, marcos institucionales y capacidades exigidas por el nuevo tipo de acción colectiva (Prats 2001, 104).

No obstante, deben diferenciarse a fines analíticos. Mientras que la gobernabilidad, definida como la "posibilidad o probabilidad de que el gobierno gobierne a su sociedad" (Aguilar 2007, 4), representa un enfoque estrictamente gubernamental (centrado en las capacidades del gobierno), el propio de la gobernanza es post-gubernamental al sostener que la dirección efectiva de la sociedad requiere -además del gobierno- de las capacidades y recursos de actores no políticos y extra-gubernamentales (Aguilar 2007, 6).

En definitiva, gobernanza y gobernabilidad se presentan como dos visiones con el mismo objetivo de restablecer la eficacia directiva de los gobiernos pero con un planteo distinto del problema que deriva lógicamente en propuestas disímiles de acción. De hecho, la gobernanza incluye a la gobernabilidad, en tanto requiere la acción de un gobierno capaz y eficaz, pero el gobierno competente es solo unos de los actores necesarios para que la dirección de la sociedad sea exitosa (Aguilar 2007, 4-7).

Por otra parte, la gobernanza presenta dos principales acepciones: una descriptiva y otra normativa/prescriptiva (Aguilar 2008). La primera resalta la importancia de la participación de la sociedad civil en las decisiones públicas, mientras que la segunda proclama la existencia de una forma correcta de gobernar. De esta última perspectiva deriva el concepto de buen gobierno (good governance), acogido por diversas escuelas de pensamiento e impulsado por varios organismos internacionales (Banco Mundial y OCDE, por ejemplo).

En las últimas décadas, el empleo del concepto de gobernanza así entendido se extendió a otras disciplinas e instituciones como herramienta para analizar el respectivo modelo de dirección que presentan determinados fenómenos de interés, tales como el sistema internacional (gobernanza global), la política urbana (gobernanza local), el medio ambiente (gobernanza ambiental) ${ }^{4}$, la integración regional (gobernanza multinivel) ${ }^{5}$, la cooperación internacional (gobernanza de la cooperación internacional) y, lógicamente, la seguridad (gobernanza de la seguridad).

Esta noción de gobernanza de la seguridad presenta, asimismo, distintas acepciones específicas según el ámbito geográfico al que refiere y las

\footnotetext{
${ }^{4}$ Empleado por el Programa de las Naciones Unidas para el Medio Ambiente (PNUMA).

${ }^{5}$ Receptado en la Carta de la Gobernanza Multinivel en Europa (2014).
} 
dimensiones de la seguridad que comprende. De este modo, se aplica tanto a asuntos mundiales (gobernanza de la seguridad internacional o global) y regionales (gobernanza de la seguridad regional) como crecientemente al terreno concreto de la seguridad pública/ciudadana/interior (gobernanza de la seguridad pública o ciudadana $)^{6} \mathrm{y}$, en menor medida, al de la defensa nacional.

En consecuencia, la aplicación del concepto a Iberoamérica requiere primeramente reseñar el alcance de la noción de seguridad a los fines del presente trabajo, máxime considerando sus diversas connotaciones actuales en el plano político-académico y los cambios de perspectivas predominantes sobre el asunto acaecidos post 11-S (Bartolomé 2006). También deviene necesario esbozar su recepción normativa-institucional en la región, para lo que resulta indispensable identificar modelos y tendencias coexistentes en su seno.

El estudio de la seguridad experimentó profundas transformaciones desde el final de la Guerra Fría, pasando de entender al Estado como único objeto referente y a los medios militares como atributo excluyente de poder, a receptar nuevas tendencias que abogan por la consideración -entre otras cuestionesde la seguridad del individuo, la importancia de otros medios distintos del castrense (por ejemplo, poder blando e inteligente) y la existencia de amenazas sin carácter puramente militar (crimen organizado, terrorismo, etc.) (Laborie Iglesias 2011).

Una visión amplia de la seguridad engloba necesariamente ambas perspectivas. Ello implica, en consecuencia, incluir en el análisis a las dos principales políticas públicas que dan cuenta de la problemática así entendida: la defensa nacional y la seguridad pública, ciudadana o interior. Otrora estrictamente compartimentadas y ejecutadas por actores distintos (fuerzas armadas por un lado y de seguridad/policiales por otro), en la actualidad presentan crecientemente estructuras comunes de planeamiento y operación.

En esta línea, en Europa se ha extendido el empleo del concepto de seguridad nacional para denotar una visión integral de la seguridad en la que se conjugan riesgos y amenazas tradicionales (estatales-militares) y nuevas (no estatales) y donde la defensa y la seguridad ciudadana son solo algunas de las dimensiones de actuación pública para conjurarlas. Ellas se reflejan en Estrategias de Seguridad Nacional (ESN) y tienen una jerarquía superior a las políticas sectoriales, siendo por definición más amplias e interagenciales (DCAF, 2008).

Mientras que España no es una excepción a dicha tendencia europea ${ }^{7}$, en América Latina el panorama es heterogéneo. Ello en virtud de que diferentes formas de recepción normativa de las nociones de seguridad y defensa

\footnotetext{
${ }^{6}$ Para su conceptualización como gobernanza de la seguridad ciudadana ver: Elkin Velásquez (2006).

${ }^{7}$ España aprobó dos ESN consecutivas: Estrategia Española de Seguridad: una responsabilidad de todos (2011) y Estrategia de Seguridad Nacional: un proyecto compartido (2013).
} 
devinieron en múltiples modelos institucionales de gestión de las políticas públicas respectivas ${ }^{8}$. Una arista visible de tales divergencias radica en el rol asignado a las fuerzas armadas, limitadas a actuar exclusivamente frente a amenazas de tipo tradicional o presentando una competencia extendida a otras (narcotráfico, por ejemplo).

Dicha pluralidad de perspectivas es explícita, por ejemplo, en el marco de los países que integran el Consejo de Defensa Suramericano, instancia creada en 2008 por los 12 Estados de América del Sur que conforman la Unión de Naciones Suramericanas (UNASUR). De hecho, fue necesario encargar a su Centro de Estudios Estratégicos de la Defensa (CEED) la confección de un informe que permita alcanzar definiciones compartidas de seguridad y defensa a pesar de las diferencias de enfoque político y doctrinario existentes entre sus miembros ${ }^{9}$.

En conclusión, describir la gobernanza de la seguridad en Iberoamérica implica para este trabajo identificar y caracterizar los mecanismos de dirección de la problemática de la seguridad existentes en torno a la arquitectura de cooperación regional de la Comunidad Iberoamericana, partiendo de una visión amplia de la seguridad que incluye a la seguridad ciudadana y a la defensa nacional. Este diagnóstico, a realizarse entre los dos próximos apartados, sentará las bases para la posterior presentación de nuevas perspectivas de cooperación en la materia.

\section{Cooperación iberoamericana}

De la primera Cumbre Iberoamericana de Jefes de Estado y de Gobierno, celebrada en Guadalajara (México) en 1991, el sistema iberoamericano ha estado -aunque con altibajos- en permanente movimiento. De todos modos, sus inicios deben remontarse décadas atrás, constituyendo una ineludible referencia fundacional la creación en 1949 de la actual Organización de Estados Ibero-americanos para la Educación, la Ciencia y la Cultura (OEI), erigida posteriormente como organismo intergubernamental en 1954.

En la actualidad se apoya como brazo ejecutivo en la Secretaría General Iberoamericana (SEGIB), creada en 2005 como organismo internacional sobre la base de su antecesora Secretaría de Cooperación Iberoamericana (SECIB) (originada en la IX Cumbre de la Habana de 1999). Su misión consiste en apoyar la preparación de las mencionadas Cumbres y ejecutar sus mandatos, expresados desde su inicio en el formato de Declaraciones y más recientemente en Programas de Acción.

\footnotetext{
${ }^{8}$ Para mayor información sobre tipos de recepción normativa y modelos institucionales en América Latina ver: Ugarte (2001), Centro de Altos Estudios Nacionales (2013) y Rial (2014).

${ }^{9}$ Informe Preliminar del CEED al Consejo de Defensa Suramericano acerca de los términos de referencia para los conceptos de seguridad y defensa en la región suramericana (2011).
} 
También lo componen, además de la SEGIB y la OEI, el Organismo Internacional de Juventud para Iberoamérica (OIJ), la Organización Iberoamericana de Seguridad Social (OISS), la Conferencia de Ministros de Justicia de los Países Iberoamericanos (COMJIB) y, desde 2015, el Comité de Dirección Estratégica de los Organismos Iberoamericanos (CoDEI). Este último tiene la función de construir sinergias y eficiencias a partir de la consolidación de la planificación estratégica y demás aspectos comunes entre las mencionadas instituciones.

Complementariamente, dispone de otros actores con competencias más específicas y menor relevancia institucional comparada. En esta dirección, y en virtud de los objetivos del análisis, se destacan la Conferencia Iberoamericana de Ministras y Ministros de Administración Pública y Reforma del Estado y el Centro de Formación e Innovación en Políticas Públicas (CEFIPP). Asimismo, se nutre del apoyo de la sociedad civil organizada en redes, como es el caso de la Red Iberoamericana de Estudios Internacionales (RIBEI).

Su actual agenda de trabajo está plasmada en el denominado Plan de Acción Cuatrienal de la Cooperación Iberoamericana 2015-2018 (PACCI). A través de ese documento, por un lado, se define como componentes transversales principales al fortalecimiento de la propia estructura de cooperación y a la modalidad de cooperación sur-sur y, por otro, se cataloga como áreas sectoriales prioritarias (denominadas Espacios Iberoamericanos) al conocimiento (EIC), la cultura (ECI) y la cohesión social (EICS).

Se subraya también el interés del sistema por impulsar la participación e innovación del individuo en la administración de los asuntos públicos, integrando así capacidades públicas, privadas y ciudadanas en su dirección -en fina sintonía con los postulados y propuestas de la reseñada perspectiva de la gobernanza-. La Carta Iberoamericana de Participación Ciudadana en la Gestión Pública (2009) representa quizá el ejemplo más acabado de iniciativa programática en esta línea.

Relegada a un segundo plano, la cuestión de la seguridad ha sido abordada parcial e intermitentemente desde diversos organismos con competencia específica en otros temas. Tal es el caso de su dimensión judicial por medio de la COMJIB, de su vertiente vial por la Conferencia Iberoamericana de Ministras, Ministros y Altos Representantes del Transporte e Infraestructura y de su impacto urbano a través del Foro Iberoamericano sobre Seguridad Ciudadana, Violencia y Políticas Públicas en el ámbito local.

Además de dicho tratamiento institucional, la seguridad ha sido objeto de distintas declaraciones en el marco de las sucesivas cumbres presidenciales. Concretamente, el apoyo a la lucha contra el terrorismo y el combate a la delincuencia organizada transnacional han sido objeto excluyente de 
Comunicados Especiales desde 2005, reafirmándose en cada caso la voluntad política por reforzar las capacidades nacionales existentes e incrementar las instancias de cooperación destinadas a prevenir y repeler ambas amenazas.

Párrafo aparte merece el Comunicado Especial sobre Seguridad Ciudadana y Seguridad Pública, realizado en el contexto de la XXI Cumbre Iberoamericana (2011). A pesar de ser una declaración extraordinaria sin carácter vinculante constituye el principal antecedente de intervención del sistema en la materia. Sus objetivos primarios fueron resaltar la importancia de planificar políticas públicas afines, introducir reformas legales y orgánicas, afianzar la cooperación internacional y fortalecer la formación de recursos humanos.

Esta proto-institucionalidad de la seguridad en la arquitectura de cooperación iberoamericana se completa con el aporte realizado por la sociedad civil formalmente vinculada al sistema, como es el caso de la ya mencionada RIBEI. No obstante, dicha red tiene su foco en los asuntos internacionales en general más que en la problemática específica de la seguridad. Análogo es el caso del Centro Latinoamericano de Administración para el Desarrollo (CLAD), cuyo énfasis está puesto en la reforma del Estado y la modernización de la administración pública.

Para algunos autores (Raggio Cachinero 2007) la falta de voluntad política por desarrollar una dimensión de la seguridad en el sistema iberoamericano se debe a la presencia superpuesta de otros esquemas de cooperación regional más consolidados (el interamericano, por ejemplo), la preferencia suramericana por canalizar el tratamiento de estos tópicos en esquemas propios de cooperación ${ }^{10}$ y la falta de percepción de algunos países iberoamericanos sobre la utilidad del régimen para dar cuenta de soluciones en la temática.

De este modo, el contraste con otros espacios de cooperación conformados por los mismos miembros resulta evidente, como ser la Organización de los Estados Americanos (OEA) y la Unión Europea (UE). Salvando lógicas diferencias de alcance y desarrollo institucional, ambos espacios-interamericano y europeo- presentan instancias orgánicas específicamente competentes en seguridad (por ejemplo, la Secretaría de Seguridad Multidimensional en la OEA y la Agencia Europea de Defensa en la UE) y una prolífica comunidad epistémica especializada.

${ }^{10}$ Tal es el caso del Consejo de Defensa Suramericano (UNASUR) dado el desarrollo organizacional alcanzado y las iniciativas programáticas implementadas (Registro Suramericano de Gastos de Defensa por ejemplo). También el MERCOSUR y la Comunidad Andina de Naciones (CAN) poseen instancias y líneas de trabajo específicas en el tema. 


\section{Seguridad en Iberoamérica}

A pesar de la escasa atención brindada a la temática desde las instituciones iberoamericanas la región ostenta una nutrida agenda de seguridad. Un repaso sobre su contenido según fuentes oficiales y académicas evidencia la presencia de tópicos de interés común a ambos lados del Atlántico, aunque fundamentalmente también de dinámicas propias de alcance subregional y nacional. Para su cabal diagnóstico se analizarán consecuentemente sendas perspectivas, además de abordajes teóricos y tendencias predominantes.

Mientras que el sistema iberoamericano se limitó simplemente a reconocer al terrorismo y al crimen organizado como temas de interés político regional, el sector académico ha ido más allá, aportando reflexiones sobre problemáticas subregionales, amenazas puntuales y dinámicas locales. El Instituto Universitario Gutiérrez Mellado (IUGM), el Instituto Español de Estudios Estratégicos (IEEE) y el Centro Superior de Estudios de la Defensa Nacional (CESEDEN) han sido históricamente las principales usinas generadoras de material al respecto ${ }^{11}$.

En general estos trabajos han sido enfocados desde la disciplina de las relaciones internacionales (contextos estratégicos, problemas de seguridad, niveles de integración, estrategias de cooperación, etc. $)^{12}$, la política comparada (entre subregiones, coyunturas temporales y casos nacionales) ${ }^{13} \mathrm{y}$, en menor medida, desde la economía de la defensa (presupuesto e industria militar), siendo por ende pocos los concebidos específicamente desde el campo del análisis de políticas públicas ${ }^{14}$.

Un somero diagnóstico de la realidad iberoamericana permite definir a la región como un espacio geoestratégico con características propias, constituyendo una de las zonas con menor conflictividad entre Estados, sin armas nucleares, con bajo gasto militar, sin amenazas que afecten el orden/estabilidad mundial pero no exenta de violencia (Fuente Cobo 2014: 2). En este marco general, el estado de su agenda de seguridad presenta concretas diferencias entre España y América Latina y el Caribe que corresponde bosquejar.

Estos contrastes inter-subregionales, sustentados en distintas lógicas de organización, capacidades y cultura estratégica, se manifiestan -entre múltiples cuestiones- en la priorización de desafíos, amenazas y riesgos, en el origen geopolítico de los problemas centrales de seguridad y en la presencia/ausencia

\footnotetext{
11 Son de especial relevancia sus publicaciones regulares: Actas de las Jornadas de Estudios de Seguridad (IUGM), Cuadernos de Estrategia (IEEE y IUGM) y Documentos de Seguridad y Defensa (CESEDEN).

${ }^{12}$ Por ejemplo ver IEEE e IUGM (2012 y 2014).

13 Por ejemplo ver: Moreyra (2011).

14 Por ejemplo ver: IUGM (2008).
} 
de compromisos supranacionales en el área. Se enumeran sucintamente a continuación a título ilustrativo:

-El terrorismo internacional (yihadista) es la principal amenaza declarada en España ${ }^{15}$, de baja trascendencia real en una América Latina donde se identifica al narcotráfico (crimen organizado) como problema transnacional de seguridad prioritario ${ }^{16}$.

-La inseguridad ciudadana (violencia y delito) es prioridad indiscutible en la agenda pública de América Latina y el Caribe ${ }^{17}$ (también el debate centrado en las capacidades idóneas para enfrentarla $)^{18}$, no revistiendo dicha trascendencia en España.

-El origen de los principales problemas de seguridad en España es externo (norte de África y Medio Oriente) ${ }^{19}$. América Latina, autodefinida como zona de $\mathrm{paz}^{20}$, no identifica amenazas extra-regionales colectivas que impliquen el uso de la fuerza.

-España es miembro de organizaciones regionales (OTAN y UE) que condicionan sus decisiones de política exterior sobre seguridad (participación en misiones multilaterales, producción común de sistemas de armas, etc.), limitantes no existentes en América Latina y el Caribe.

Es menester aclarar que bajo dicha caracterización de Latinoamérica y el Caribe subyacen diferencias de impacto y priorización de los problemas de seguridad y, lógicamente, de capacidades estatales para enfrentarlos. También el grado de cooperación subregional alcanzado en la materia es sustantivamente disímil. Ergo, todo estudio detallado de su agenda común de seguridad debe contemplar los contrastes existentes entre México, Centroamérica, el Caribe y Suramérica ${ }^{21}$ (no siendo el objetivo del presente trabajo).

Para complementar dicha reseña de la coyuntura regional debe puntualizarse que, en ausencia de instituciones iberoamericanas competentes, las iniciativas de cooperación entre España y América Latina en seguridad se instrumentaron históricamente a través de canales bilaterales. Entre los principales temas de interés gestionados bajo esta modalidad se destacan la venta de productos elaborados por el sector industrial militar español

\footnotetext{
${ }^{15}$ Según se desprende de la Estrategia de Seguridad Nacional: un proyecto compartido (2013).

${ }^{16}$ Sobre este último punto ver: Alda Mejías (2011).

17 Para mayor información ver: Programa de las Naciones Unidas para el Desarrollo (PNUD) (2013).

${ }^{18}$ Se identifica una sostenida tendencia hacia la militarización de la seguridad pública, situación que conduce a un progresivo policiamiento de las misiones de las Fuerzas Armadas. Para complementar dicho diagnóstico ver: Rojas Aravena (2012).

19 Para mayor información ver: Fernández (2015).

${ }^{20}$ Según la Proclama de América Latina y el Caribe como Zona de Paz elaborada por la Comunidad de Estados Latinoamericanos y Caribeños (CELAC) en la II Cumbre de La Habana (2014) y otros documentos análogos de la UNASUR, la CAN y del MERCOSUR.

${ }^{21}$ Para mayor análisis ver: Cheyre (2015).
} 
en América Latina y la formación de civiles y militares en instituciones españolas (Bartolomé 2015b).

De cara al fortalecimiento de una agenda positiva, que potencie y diversifique tales canales de cooperación, pueden aprovecharse las similitudes generadas por la analogía de diseño de las estructuras burocrático-legales que la mayoría de los países latinoamericanos tiene en relación a las de la península ibérica, fruto lógicamente de cimientos comunes. Indudablemente, España y Portugal han sentado las bases de una cosmovisión del estado y del derecho público que aún subsisten (aunque en tensión) en América Latina.

En esta línea reviste especial trascendencia un fenómeno escasamente considerado por los hacedores de políticas de cooperación regional como es la coincidencia de procesos internos de reforma y modernización del sector seguridad acaecidos en América Latina en la última década. A pesar de tener jerarquías opuestas en la escala de prioridades de las agendas políticas nacionales, el diagnóstico incluye a las áreas estatales competentes en seguridad ciudadana y defensa.

En seguridad ciudadana estos cambios respondieron, entre múltiples causas, a la necesidad de adaptar las instituciones responsables ante el incremento y complejidad del delito -siendo una demanda social prioritaria ${ }^{22}$. Simultáneamente, en el ámbito de la defensa las modificaciones se debieron, mayormente, a la voluntad de reforzar la conducción política de un sector postergado en la agenda gubernamental de las primeras décadas post recuperación de la democracia ('80-'90) $)^{23}$.

La cuestión de la administración de la política de seguridad constituye así un objeto de interés común y, por ende, una oportunidad concreta de cooperación para el sistema iberoamericano en sus modalidades sur-sur y norte-sur. Ello último en la inteligencia de que la experiencia española, tanto a nivel local como multilateral, puede ser de suma utilidad en América Latina, al igual que determinadas mejores prácticas extraídas de organizaciones regionales y casos nacionales latinoamericanos pueden serlo en España ${ }^{24}$.

\section{Perspectivas de cooperación}

Cierto es que el sistema iberoamericano ha evitado inmiscuirse en asuntos de seguridad desde un enfoque comprehensivo, no habiendo desarrollado un andamiaje institucional específico acorde a la actual trascendencia del tema en las agendas públicas de sus miembros. También es real que el sector académico

\footnotetext{
${ }^{22}$ Para mayor información ver: Frühling (2003).

${ }^{23}$ Para mayor información ver: Consejo de Defensa Suramericano (2010a).

${ }^{24}$ Por ejemplo, sobre transparencia de gastos de defensa en el marco de la UNASUR (Registro Suramericano de Gastos de Defensa).
} 
especializado ha abordado el fenómeno, en gran medida, desde visiones distintas del análisis de políticas públicas (focalizado por ejemplo en su planeamiento, gestión y evaluación).

Sin embargo, la descripta coyuntura de reformas sectoriales sobrevenidas en el último decenio genera las condiciones adecuadas para incorporar a la administración de la política de seguridad como nuevo eje transversal de interés en el ámbito iberoamericano, instalándose como objetivo programático de sus organismos la identificación de alternativas de mejora de su calidad y resultados. El intercambio de experiencias subregionales sería un primer paso para generar valor agregado en este camino.

Es válido puntualizar que esta iniciativa encuentra respaldo institucional, por un lado, en la catalogación de la cohesión social como área prioritaria dentro del PACCI 2015-2018 (dada la intrínseca relación entre seguridad y desarrollo) y, por otro, en el declarado compromiso de las diversas plataformas del régimen con la participación ciudadana y la calidad de las políticas públicas. Desde otra perspectiva, redundaría también en un empoderamiento per se del propio sistema y en un decidido impulso al modelo de cooperación sur-sur que promueve.

Concretamente, podrían incluirse líneas de acción sobre desafíos contemporáneos en administración de la seguridad tales como: diseño organizacional, planeamiento estratégico, gestión por resultados, integración logística, I+D $+\mathrm{I}$, planificación presupuestaria, formulación y evaluación de proyectos, conjuntez operacional, educación de funcionarios civiles, formación profesional, gestión de la información, adiestramiento conjunto, operaciones combinadas y gestión multilateral de programas de armamento y material ${ }^{25}$.

Asimismo, en el plano académico/educativo la temática podría ser agregada a los programas del Centro de Formación e Innovación en Políticas Públicas (CEFIPP) y del Centro de Altos Estudios Universitarios (CAEU) de la OEI. Análoga reflexión podría aplicarse al Centro Latinoamericano de Administración para el Desarrollo (CLAD) y demás think thanks adscriptos al sistema. De hecho, este debería contar con el apoyo formal (vía inscripción en el registro respectivo) de una red iberoamericana específicamente dedicada al tema.

En el plano organizacional, algunas de las iniciativas (no excluyentes) a encarar para la instalación de la problemática de la administración de la seguridad en el ámbito iberoamericano de cooperación podrían ser las siguientes:

-Identificación del fortalecimiento institucional de las esferas de gestión pública de la seguridad como programa dentro del Espacio Iberoamericano de Cohesión Social (EICS).

${ }^{25}$ Este último punto se refiere a los programas de pooling \& sharing, estandarización de material y consolidación de demandas de bienes y servicios comunes de la Agencia Europea de Defensa (EDA). 
-Inclusión de la modernización del sector seguridad como tópico de agenda de la Conferencia Iberoamericana de Ministras y Ministros de Administración Pública y Reforma del Estado.

-Reactivación del Foro Iberoamericano sobre Seguridad Ciudadana, Violencia y Políticas Públicas en el ámbito local.

-Generación de contenidos académicos específicos para funcionarios y especialistas en las plataformas educativas existentes (CEFIPP y CAEU). -Solicitud formal al CLAD de incorporar un área de trabajo específica en la materia.

Finalmente, se enumeran a continuación una serie de temas de interés que podrían nutrir el plan de acción de estos organismos, extraídos de las conclusiones emanadas de la Mesa Redonda No 1 (2016) de la iniciativa Pensado la Seguridad de Iberoamérica, organizada por la Universidad del Salvador (USAL) y el Grupo de Estudios Internacionales (GESI) de la Universidad de Granada (UGR), a saber:

-Utilidad de analizar los esquemas de articulación regional en seguridad en América Latina y Europa en formato de mapa comparado.

-Recomendación de intercambiar lecciones aprendidas y mejores prácticas sobre planeamiento y articulación de las políticas de seguridad y defensa, principalmente entre España y Suramérica.

-Inclusión de los recursos naturales como tema de análisis de la agenda de seguridad regional.

-Identificación de la relación entre seguridad y desarrollo como prioridad y condicionante de la agenda de seguridad en la región.

-Consideración de la tecnología ( $\mathrm{I}+\mathrm{D}+\mathrm{I})$ como problema de la agenda de seguridad regional.

-Importancia de compartir lecciones aprendidas sobre el proceso de creación e implementación de los Consejos de Defensa (CDS) y de Seguridad Ciudadana de la UNASUR.

-Incorporación del análisis comparado de la evolución presupuestaria reciente (contenido y calidad) en el área seguridad como tema prioritario de investigación académica y asistencia técnica inter-subregional.

-Trascendencia del Registro Suramericano de Gastos de Defensa de la UNASUR como caso de estudio y utilidad como herramienta de cooperación para extender a nivel regional.

-Instalación de las fuerzas intermedias de seguridad como tema de interés para intercambiar mejores prácticas entre países y subregiones.

-Exploración de las diferencias conceptuales intra-regionales entre seguridad y defensa en el plano del derecho internacional (actores y nuevas amenazas). 
-Impulso del intercambio de metodologías y mejores prácticas para la gestión de proyectos regionales de integración logística en seguridad, recurriendo a la experiencia multilateral española y a la propia europea (ej. EDA).

\section{Consideraciones finales}

Existe una deuda histórica del sistema iberoamericano con la administración pública de la seguridad, situación que reviste actualmente especial trascendencia dada la centralidad del tema en la consideración de sus gobiernos y ciudadanía. La construcción de una gobernanza iberoamericana de la seguridad, multidimensional y receptiva hacia el aporte de capacidades sociales, ha sido hasta ahora embrionaria, encontrándose solo antecedentes de abordajes parciales y pronunciamientos genéricos, esporádicos y no vinculantes.

$\mathrm{Su}$ incipiente nivel de desarrollo institucional es evidente comparado con otros esquemas análogos de cooperación, principalmente el interamericano y el europeo, siendo incluso válida la contraposición con el reciente proceso de cooperación suramericano (UNASUR), prolífico en la concertación de políticas sectoriales y la generación de programas de cooperación en el marco de sus Consejos de Defensa y de Seguridad Ciudadana, Justicia y Coordinación de Acciones contra la Delincuencia Organizada.

No obstante, la desaceleración en la que se encuentra sumida hoy la UNASUR, sumada al incierto futuro de la Unión Europea como modelo de integración y a la declamada reticencia de la Administración Trump por auspiciar regímenes internacionales de cooperación política en general (que afecta incluso a la OTAN) crea las condiciones para que sea el propio sistema iberoamericano el que canalice-al menos parcialmente-intereses y necesidades sobre temáticas hasta ahora desatendidas por éste como la seguridad.

Perspectivas globales y coyuntura regional convergen así generando una clara oportunidad para deconstruir viejas tendencias y apostar por la implementación de una agenda iberoamericana de la seguridad que multiplique las alternativas de cooperación existentes (incluso de naturaleza sur-sur), contribuya a dinamizar el funcionamiento del sistema en su conjunto incorporando el aporte de la sociedad civil e incremente su legitimidad de resultado al involucrarse en la atención de una indudable prioridad cotidiana para la ciudadanía.

En consecuencia, el desafío es edificar una agenda positiva en seguridad focalizada en la generación de líneas concretas de cooperación sobre necesidades comunes de gestión pública. Su implementación estaría en manos de los organismos ya existentes, a partir de los cuales se impulsaría el intercambio 
de lecciones aprendidas, mejores prácticas y la creación de programas de formación académica y profesional. Ello tendría efectos palpables de corto plazo sin impactos presupuestarios ni reestructuraciones organizativas de magnitud.

En un contexto signado por el aumento de la conflictividad, la emergencia de posturas aislacionistas materializadas incluso en muros físicos y simbólicos, el avance de movimientos conservadores en diferentes partes del mundo y la erosión del multilateralismo a nivel global, el resurgir del iberoamericanismo puede imprimir nuevo oxígeno a ambos lados del Atlántico, sentando las bases para la proyección de vínculos que devengan en soluciones innovadoras a problemas complejos.

Mientras el sistema internacional comienza apresuradamente a encerrarse en sí mismo, apostar al multilateralismo y a la generación de políticas públicas regionales transformadoras parece una tarea difícil de concretar. No obstante, es en tiempos de crisis y austeridad como el presente cuando la conjunción de gobierno, sociedad y ciudadanía (lo público y lo privado) tiene mayor responsabilidad de ser creativa, debiendo sinérgicamente invertir tiempo y energía en fundar nuevos caminos. Eso sí, con agendas propias y no a través de la implementación de problemáticas importadas, funcionales a sectores que sólo buscan incrementar sus propias ganancias individuales. 


\section{Referencias bibliográficas:}

Agencia Europea de Defensa (EDA), EDA's pooling \& sharing, EDA, 2013. Aguilar Villanueva, L., El aporte de la política pública y de la Nueva Gestión Pública a la gobernanza, Caracas, CLAD, Revista Reforma y Democracia $\mathrm{N}^{\circ} 39$ (2007).

Gobernanza y gestión pública, México, Fondo de Cultura Económica (2008). Alda Mejías, S., Fortalecimiento estatal y cooperación multilateral: factores imprescindibles contra el narcotráfico en América Latina, Instituto Español de Estudios Estratégicos (IEEE), Documento de Opinión $\mathrm{N}^{\circ}$ 43/2011 (2011).

Alda, S. y Gómez, V. (editores), El concepto y las relaciones multilaterales de seguridad y defensa en el contexto de la UNASUR, Madrid, Instituto Universitario Gutiérrez Mellado y Ministerio de Defensa Nacional de Ecuador, 2012.

Arriola, S., Garranzo, R. y Ruiz Jiménez, L. (coord.), La renovación de la cooperación iberoamericana: transformaciones para una agenda post2015, Madrid, Secretaría General Iberoamericana (SEGIB) - Agencia Española de Cooperación Internacional para el Desarrollo (AECID), 2013.

Arteaga, F., Propuesta para la implementación de una estrategia de seguridad nacional en España, Real Instituto Elcano. Documento de Trabajo No 19/2011 (2011).

- La defensa que viene: criterios para la reestructuración de la defensa en España, Real Instituto Elcano, Elcano Policy Paper No 3/13 (2013).

Arteaga, F. y Fojón, E., El planeamiento de la política de defensa y seguridad en España, Madrid, Instituto Universitario Gutiérrez Mellado (IUGM), 2007.

Ballesteros Martín, M., La política común de seguridad y defensa tras el Consejo Europeo de diciembre 2013, Instituto Español de Estudios Estratégicos, Documento de Análisis No 03/14 (2014).

Bartolomé, M., La seguridad internacional post 11S: contenidos, debates y tendencias, Buenos Aires, Instituto de Publicaciones Navales, 2006.

- Relaciones entre España y América Latina en el ámbito de la defensa, Revista de Relaciones Internacionales, Defensa y Seguridad, vol. N 10 (2015a), págs. 111-131.

- La cooperación entre España e Iberoamérica en el campo de la defensa: dos casos de estudio, Revista Enfoques: Ciencia Política y Administración Pública, vol. XIII, Nº 23 (2015b), págs. 31-48.

Beliz, G., Gobernar la seguridad ciudadana en América Latina y el Caribe: amenazas, desafios y nudos estratégicos de gestión, Banco Interamericano de Desarrollo, Documento de Debate N IDB-DP-215 (2012). 
Bringel, B y Cairo Carou, H., Articulaciones del Sur Global: afinidad cultural, internacionalismo solidario e Iberoamerica en la globalización contrahegemónica, Geopolítica(s), vol. 1, Nº 1 (2010), págs. 41-63.

Bucur-Marcu, H. et al (editores), Gestión de la defensa: una introducción, Ginebra, Centro de Ginebra para el Control Democrático de Fuerzas Armadas (DCAF), 2013.

Centro de Altos Estudios Nacionales, Conceptos de seguridad y defensa de los países iberoamericanos: desde la óptica de sus Colegios de Defensa, Montevideo, 2013.

Centro de Estudios Estratégicos de Defensa, Institucionalidad de la defensa en Suramérica, Buenos Aires, Consejo de Defensa Suramericano (CEED), 2015.

Centro Superior de Estudios de la Defensa Nacional, Hacia una política de cooperación en seguridad y defensa con Iberoamérica, Madrid, CESEDEN, Monografías del CESEDEN N 84, 2006.

- Seguridad y defensa en Iberoamerica: posibilidades actuales para la cooperación, Madrid, Documentos de Seguridad y Defensa $\mathrm{N}^{\circ} 15$, CESEDEN, 2007.

- La creación de UNASUR en el marco de la seguridad y la defensa, Madrid, Documentos de Seguridad y Defensa N²9, CESEDEN, 2010.

- España y su posible papel en las iniciativas de "partenariado" iberoamericano en OTAN e iniciativas CSDP en la UE, Madrid, Documentos de Seguridad y Defensa $\mathrm{N}^{\circ}$ 24, CESEDEN, 2013.

Cheyre, J., Las amenazas a la seguridad en América Latina, Georgetown University, Revista Globalización, Competitividad y Gobernabilidad, vol. 9, $\mathrm{N}^{\circ} 1$ (2015), págs. 51-73.

Comini, N., El origen del Consejo de Defensa Suramericano: modelos en pugna desde una perspectiva argentina, Revista de Estudios en Seguridad Internacional, vol. $1 \mathrm{~N}^{\mathrm{o}} 2$ (2015).

Comini, N. y Frenkel, A., Un lustro de UNASUR: balance y perspectivas del proceso de integración sudamericano, Universidad Nacional de La Plata (2014).

Comini, N., Jordán, J. y Vega, J., Pensando la seguridad de Iberoamérica, Universidad del Salvador (USAL) y Grupo de Estudios en Seguridad Internacional (GESI), Boletín Informativo Nº1 (2016).

Consejo de Defensa Suramericano (CDS), Modernización de Ministerios de Defensa, Consejo de Defensa Suramericano - Presidencia Pro Témpore Ecuador, Cuadernos de Defensa No 1, 2010a.

- Confianza y Seguridad en América del Sur, Consejo de Defensa Suramericano - Presidencia Pro Témpore Ecuador, Cuadernos de Defensa No 2, 2010b. 
- Informe Preliminar del CEED al Consejo de Defensa Suramericano acerca de los términos de referencia para los conceptos de seguridad y defensa en la región suramericana, Centro de Estudios Estratégicos de la Defensa, 2011.

Crozier, M., Huntington, S. y Watanuki, J., The crisis of democracy: report on the governability of democracies to the Trilateral Commission, Nueva York, New York University Press, 1975.

Dussel, E., Eurocentrismo y modernidad (Introducción a las lecturas de Frankfurt), [en Mignolo, W. (compilador): Capitalismo y geopolítica del conocimiento: el eurocentrismo y la filosofía de la liberación en el debate intelectual contemporáneo, Buenos Aires, Ediciones del Signo, 2001], págs. 57-70.

Elkin Velásquez, M., La gobernabilidad y la gobernanza de la seguridad ciudadana: hacia una propuesta operacional, Centro de Documentación del Programa URBAL (2006).

España. Ministerio de Defensa, Revisión estratégica de la defensa, Madrid, Ministerio de Defensa, 2003.

- European Defence Agency: past, present \& future, Madrid, Ministerio de Defensa, 2010.

- Estrategia Española de Seguridad: una responsabilidad de todos, Madrid, Catálogo de Publicaciones de la Administración General del Estado, 2011.

- Estrategia de Seguridad Nacional: un proyecto compartido, Madrid, Catálogo de Publicaciones de la Administración General del Estado, 2013.

Fernández, H., España frente a los restos en el Magreb y Oriente Medio en 2015, Real Instituto Elcano, ARI 12/2015 (2015).

Frühling, H., Policía comunitaria y reforma policial en América Latina: ¿cuál es el impacto?, Santiago, Centro de Estudios en Seguridad Ciudadana (Universidad de Chile), Serie Documentos (2003).

Fuente Cobo, I., Análisis crítico de la seguridad e Iberoamérica, Instituto Español de Estudios Estratégicos, Documento de Análisis No 29/14 (2014).

Geneva Centre for the Democratic Control of Armed Forces (DCAF), Politicas de seguridad nacional, Ginebra, DCAF, Series de Fichas del DCAF sobre Buen Gobierno y Reforma del Sector de la Seguridad N 2 (2008).

Gonzalo Navarro V., Marco estratégico de la Agencia Europea de Defensa: nuevas iniciativas operativas, Instituto Español de Estudios Estratégicos, Documento de Opinión del IEEE Nº 61/2011 (2011).

Gratius S., ¿Porqué España no tiene una política hacia América Latina?, Policy Brief $\mathrm{N}^{\circ}$ 14, FRIDE (2010).

Instituto Español de Estudios Estratégicos (IEEE), Valores, principios y seguridad en la Comunidad Iberoamericana de Naciones, Madrid, Cuadernos de Estrategia $N^{\circ} 126$ (2003). 
Instituto Español de Estudios Estratégicos (IEEE) e Instituto Universitario Gutiérrez Mellado (IUGM), Iberoamérica: nuevas coordenadas, nuevas oportunidades, grandes desafios, Madrid, Cuadernos de Estrategia $N^{\circ} 136$ (2007).

- Los desafios de la seguridad en Iberoamérica, Madrid, Cuadernos de Estrategia $\mathrm{N}^{\circ} 158,2012$.

- Cooperación con Iberoamérica en materia de defensa, Madrid, Cuadernos de Estrategia $\mathrm{N}^{\circ} 1171,2014$.

Instituto Universitario Gutiérrez Mellado (IUGM), Seguridad humana y nuevas políticas de defensa en Iberoamérica, Madrid, IUGM, 2007.

- La administración de la defensa en América Latina, Tomos I, II y III, Madrid, 2008.

- Guía del espacio iberoamericano de paz, seguridad y defensa, Madrid, 2010.

- Actas IV Jornadas de Estudios de Seguridad, Madrid, IUGM, 2012, págs. 465-954.

- Actas V Jornadas de Estudios de Seguridad, Madrid, IUGM, 2013, págs. 671-1002.

- Actas VI Jornadas de Estudios de Seguridad, Madrid, IUGM, 2014, págs. 495-894.

- Actas VII Jornadas de Estudios de Seguridad, Madrid, IUGM, 2015, págs. 435-772.

Instituto Universitario Gutiérrez Mellado (IUGM) y Ministerio de Defensa Nacional de Ecuador, El concepto y las relaciones multilaterales de seguridad y defensa en el contexto de la UNASUR, Madrid, IUGM, 2012.

Jordán Enamorado, J., Enfoques teóricos de los estudios estratégicos [en Jordán

Enamorado, J. (coord.): Manual de estudios estratégicos y seguridad internacional, Madrid, Plaza y Valdez Editores, 2013], págs.15-43.

Laborie Iglesias, M., La evolución del concepto de seguridad, Instituto Español de Estudios Estratégicos (IEEE), Documento Marco No 5/2011 (2011).

Magariños, G., Integración económica latinoamericana: proceso ALALC/ ALADI 1950-2000, Montevideo, ALADI, 2005.

Morán López, F., Prólogo [en Segunda Cumbre Iberoamericana, Madrid, España, Julio 1992. Discursos y Documentos, México, Fondo de Cultura Económica, 1993].

Moreyra, M. (editor), Política de defensa en América Latina, Ginebra, Centro de Ginebra para el Control Democrático de las Fuerzas Armadas (DCAF), 2011.

Natera Peral, A., La gobernanza como modo emergente de gobierno y gestión pública, Instituto Nacional de Administración Pública, Revista Gestión y Análisis de Políticas Públicas (GAPP), N 33-34 (2005). 
Organización de los Estados Americanos, Documentos claves de la OEA sobre seguridad Volumen 1: Seguridad Nacional, OEA ( $\sin$ año).

Organización de los Estados Iberoamericanos (OEI), Memoria 2014-2015, OEI, 2016.

- Programa Presupuesto 2016, OEI, 2016.

Orozco, G., El concepto de seguridad en la teoría de las relaciones internacionales, Barcelona, Revista CIDOB dÁffers Internacionals $\mathrm{N}^{\mathrm{o}} 72$ (2006).

Peña, J., Políticas públicas de defensa, [en Jordán Enamorado, J. (coord.): Manual de estudios estratégicos y seguridad internacional, Madrid, Plaza y Valdez Editores, 2013], págs. 239-264.

Prats i Català, J., Gobernabilidad democrática para el desarrollo humano: marco conceptual y analitico, Institut Internacional de Governabilitat de Catalunya, Revista Instituciones y Desarrollo, N 10 (2001), págs. 103148.

Programa de las Naciones Unidas para el Desarrollo (PNUD), Seguridad ciudadana con rostro humano: diagnóstico y propuestas para América Latina, PNUD, 2013.

Raggio Cachinero, B., La cooperación de defensa iberoamericana [en Instituto Español de Estudios Estratégicos e Instituto Universitario Gutiérrez Mellado: Iberoamérica: nuevas coordenadas, nuevas oportunidades, grandes desafios, Madrid, Cuadernos de Estrategia $\mathrm{N}^{\circ}$ 136, 2007], pág. 195-224.

Revista Española de Defensa, Cooperación con América, Revista Española de Defensa, $\mathrm{N}^{\mathrm{o}}$ noviembre (2014), págs. 48-49.

Rial, J., América Latina y sus problemas de seguridad y defensa: incertidumbre en tiempos de cambio constante [en Instituto Español de Estudios Estratégicos e Instituto Universitario Gutiérrez Mellado: Cuadernos de Estrategia No 71: Cooperación con Iberoamérica en materia de defensa, IEEE y IUGM, 2014], págs. 31-55.

Rojas Aravena, F., Seguridad internacional, el espacio y posición de América Latina [en Instituto Español de Estudios Estratégicos (IEEE) e Instituto Universitario Gutiérrez Mellado (IUGM): Los desafíos de la seguridad en Iberoamérica, Madrid, Cuadernos de Estrategia $\left.\mathrm{N}^{\circ} 158,2012\right]$, pág. 13-75.

América Latina: cooperación en seguridad y defensa en un contexto de cambios globales, Araucaria Revista Iberoamericana de Filosofía, Política y Humanidades, Año 16, Nº 32 (2014).

Sanahuja, J. y Verdes-Montenegro Escánez, F., Seguridad y defensa en Suramérica: regionalismo, cooperación y autonomía en el marco de la UNASUR, CRIES, Anuario de Integración, N 10 (2014), págs. 487-530. 
Secretaría General Iberoamericana (SEGIB), Plan de Acción de la Cooperación Iberoamericana 2015-2018, SEGIB, sin año.

Seitz, A., Integración Latinoamericana: caminos, dilemas y desafios. Red para la Integración Latinoamericana (REDILA) (2010).

Ugarte, J., Los conceptos de defensa y seguridad en América Latina: sus peculiaridades respecto de los vigentes en otras regiones y las consecuencias politicas de tales peculiaridades, Latin American Studies Association (2001).

Vega, J., Integración logística regional en el marco del Consejo de Defensa Suramericano, Buenos Aires, Ministerio de Defensa, Cuadernos en Actualidad en Defensa y Estrategia, No 2 (2010), págs. 15-32.

- El planeamiento de la defensa en España 2008-2015: análisis de política pública, GESI, Revista de Estudios en Seguridad Internacional, vol. $1 \mathrm{~N}^{\mathrm{o}}$ 2 (2015), págs. 35-63.

Velázquez, E., La gobernabilidad y la gobernanza de la seguridad ciudadana: hacia una propuesta operacional, Colombia, Grupo Trans-disciplinario de Investigación en Ciencias Sociales, Borradores de Método 48 (2005). 